\title{
Cuando el capital pierde el maquillaje verde. Impactos de la producción de biodiesel en Santiago del Estero, Argentina
}

\author{
When capital loses its green makeup. The impact of biodiesel production \\ on Santiago del Estero Province, Argentina
}

Virginia Toledo-López ${ }^{1}$

\author{
Palabras-clave: \\ Agrocombustibles \\ Agronegocio \\ Responsabilidad social empresarial \\ Conflicto ambiental
}

\begin{abstract}
Resumen
El artículo examina el manejo ambiental en la producción de biodiesel en Santiago del Estero (Argentina), enfocándose especialmente en el tratamiento de los efluentes líquidos. Para ello se analizan, a través de una metodología cualitativa, repertorios de actores en relación con sus estrategias territoriales. El estudio observa la producción de biodiesel en tanto reafirmación del proceso de expansión del agronegocio en la región e interpreta sus impactos ambientales, en particular la contaminación de aguas y del espacio de vida de la población local, como formas de despojo. Además, cómo las prácticas materiales de los actores hegemónicos pese a enmarcarse en la perspectiva de la modernización ecológica a través de la figura de la Responsabilidad Social Empresarial, están más bien asociadas a un enfoque no preventivo y estándar de la cuestión ambiental. Finalmente, considera las productividades del conflicto social, en términos de aprendizajes y efectos positivos en el territorio.
\end{abstract}

\section{Keywords:}

Agrofuel

Agribusiness

Corporate social responsibility

Environmental conflict

\begin{abstract}
The article analyses the environmental management of biodiesel production in Santiago del Estero Province, Argentina, focusing on the treatment of effluents. Therefore, using a qualitative methodology, this article studies the main actors' repertoires concerning their territorial strategies. The production of biodiesel is considered a reaffirmation of the agribusiness expansion in the area and its environmental impact as forms of dispossession of the inhabitants. The study shows that the practices of hegemonic actors are associated with a non-preventive and standard approach to environmental issues, even when they use the ecological modernization frame through the Corporate Social Responsibility. Finally, it reflects on a positive effect of environmental conflicts in the territory.
\end{abstract}

${ }^{1}$ Consejo Nacional de Investigaciones Científicas y Técnicas (CONICET), Instituto de Estudios para el Desarrollo Social, Universidad Nacional de Santiago del Estero-CONICET, Argentina. vtoledolopez@gmail.com 


\section{INTRODUCCIÓN}

La producción de biodiesel se ha incrementado notablemente en tiempos recientes en Argentina, posicionándose hacia 2010 el país como el principal exportador y el tercer productor a nivel mundial. En tanto productores, el mercado mundial de biodiesel está liderado por Europa (especialmente Alemania), seguido de cerca por Estados Unidos, Argentina y Brasil. Por su parte, Argentina e Indonesia concentran las exportaciones, con Estados Unidos y la Unión Europea como principales importadores (OCDEFAO 2017).

En Argentina su producción se realiza a partir de soja y pasó de unas 711.864 en 2008 a unas 2.584.290 toneladas en 2014, de las cuales el $40 \%$ son destinadas al mercado interno, creado por Ley nacional desde 2010 (INDEC, 2016). En el año 2006 se sancionó la Ley de Biocombustibles, 26.093 que generó un marco legal para los emprendimientos y un mercado para su consumo a nivel nacional (desde el 2010). En sector agrícola, se estima que el $80 \%$ de la soja se destina a la elaboración de aceite y de este, el $30 \%$ es destinado a la producción de biodiesel. El 60\% del biodiesel nacional va al exterior (al igual que el 65\% del aceite de soja). Según datos del Ministerio de Hacienda (2017), la producción de soja en el país en 2016 fue de 58 millones de toneladas, obtenidas en 20 millones de has.

El dinamismo del biodiesel a principios de siglo se comprende como respuesta al impulso que a nivel global ha tenido la generación de combustibles alternativos al petróleo en un momento de altos precios de los hidrocarburos en los mercados internacionales (especialmente entre 2003 y 2008), proyecciones de escasez; en el marco de los objetivos de reducción de emisiones de gases de efecto invernadero (GEI), y en el contexto nacional de expansión del modelo del agronegocio desde mediados de los noventa, que generó las bases para su difusión (TOLEDOLÓPEZ, 2013; 2017a; 2018). Los llamados "biocombustibles" fueron impulsados como una energía renovable desde sectores del empresariado nacional y trasnacional a través de medios de comunicación masiva, en el ámbito político en planes de gobierno, programas y proyectos, y en el sistema científico nacional resaltando su condición de renovable, la reducción de GEI, su potencialidad para desplegar cadenas de valor y promover el desarrollo regional (HILBERT et al., 2012; ROZEMBERG et al., 2009; CARRIZO et al.,
2009; SCHEINKERMAN DE-OBSCHATKO; BEGENISIC, 2006), advirtiendo en algunos casos sobre otros posibles impactos en el ambiente y en la seguridad alimentaria, así como también desde la soberanía energética y alimentaria (TOLEDO-LÓPEZ, 2016ab; 2018).

Los efectos ambientales de la producción de agroenergía han sido debatidos a nivel mundial. Desde una perspectiva ambiental y social, su balance energético global, la competencia con la producción de alimentos y los impactos generados por la expansión de monocultivos que sirven como insumo son sólo algunos de los puntos que se han cuestionado en lo que podría definirse como la construcción de una cuestión ambiental en torno a los agrocombustibles. En este marco recuperamos los aportes de quienes resaltan su balance energético negativo (PIMENTEL; PATZEK, 2005), la emisión de gases efecto invernadero (PANICHELLI, 2006), la competencia con otros usos de la tierra (SMOLKER et. al., 2008) y los impactos sobre los sistemas agroalimentarios (RUNGE; SENAUER, 2007; BORRAS et al., 2010), entre otros. El presente trabajo se inscribe en esta discusión, interrogándose por los impactos y efectos en el plano local.

Se indaga específicamente en los conflictos relativos al manejo ambiental en el caso de la producción de biodiesel en Santiago del Estero, especialmente en relación al tratamiento de los efluentes desde su instalación (2009) y hasta 2012, cuando se realizó de trabajo de campo para esta investigación. En otros trabajos hemos profundizado en el análisis de la estrategia de acumulación del grupo empresario que dinamizó el proyecto (TOLEDO-LÓPEZ, 2017b) y en la dimensión simbólica de este proceso de territorialización (TOLEDO-LÓPEZ, 2016a), siendo éste análisis parte de una tesis doctoral en ciencias sociales (TOLEDO-LÓPEZ, 2016b). Interesa aquí dar cuenta de la conflictividad ambiental en torno al emprendimiento, a fin de profundizar en el análisis de los mecanismos de acumulación puestos en juego y visibilizar los límites de la Responsabilidad Social Empresarial (RSE). Siguiendo a Azuela y Mussetta (2008) se mantiene aquí una mirada sociológica sobre la cuestión ambiental, procurando ver a los conflictos como parte de un proceso de formación y transformación del orden social, y no como anomalías que deberían evitarse. De este modo, en última instancia, se pretende reflexionar sobre la "productividad del conflicto" (MERLINSKY, 2013), en el sentido de aprehender sus efectos y resultados positivos.

Para la consecución de los objetivos 
planteados se desplegó una metodología cualitativa tendiente a observar los relatos de diferentes actores vinculados al proyecto de producción de biodiesel. Se realizaron aproximadamente treinta entrevistas semi estructuradas a funcionarios públicos y referentes políticos del gobierno nacional, provincial y local, integrantes del grupo empresario, habitantes de la ciudad Frías aledaños al Parque Industrial donde se instaló la empresa, a referentes de instituciones educativas y a empleados y ex-empleados de la empresa. La selección de los individuos fue no-aleatoria, predominando la técnica de bola de nieve de Goodman (1961), a partir de las indicaciones de informantes clave, y hasta alcanzar el nivel teórico de la saturación. Estas fuentes primarias fueron complementadas con datos provenientes de fuentes secundarias (especialmente escritos periodísticos, discursos presidenciales y audiovisuales disponibles en Internet) y recorridos y observaciones en la fábrica y sus alrededores. Así se constituyó el "corpus general" de información, que fue revisado a fin de discriminar aquello que era pertinente y relevante para el problema de investigación. La sistematización y organización de ese material permitió generar el "corpus de análisis" (REGUILLO, 1998), iniciándose así la etapa de interpretación y análisis de discurso (ÍÑGUEZRUEDA, 2006). Finalmente, se estructuró un "esquema narrativo-histórico" a través de un diseño analítico-argumental (en el que se integraba el marco teórico de la investigación) buscando la relación entre dimensiones socioculturales, políticas y económicas (SAUTÚ et al., 2005).

\section{LA PRODUCCIÓN DE BIODIESEL EN FRÍAS COMO REAFIRMACIÓN DEL PROCESO DE EXPANSIÓN DEL AGRONEGOCIO EN SANTIAGO DEL ESTERO}

En el año 2006 se anunció la firma de un acta de compromiso entre el gobierno de la provincia de Santiago del Estero, a cargo del Gerardo Zamora y un grupo empresario de origen tucumano (TOLEDO-LÓPEZ, 2018). Se planeaba desplegar un mega-emprendimiento de producción de biodiesel, en sintonía con uno de los principales objetivos de la gestión provincial: el fomento al agregado de valor a la producción primaria de la región, plasmado en la Ley Provincial de Promoción Industrial, 6.750 sancionada ese mismo año. La Ley estableció un sistema de promoción y desarrollo industrial que tenía como objetivo primario "propiciar la instalación de nuevas industrias" y "la ampliación de las ya existentes" (Art. 2), a partir del "el aprovechamiento racional de los recursos e insumos de la provincia". Para ello otorgaba una serie de beneficios a las industrias "transformadoras de materias primas locales". En el Art. 3 establece como condición para acceder al sistema promocional el cumplimiento de alguna de las siguientes condiciones: uso de materia prima de origen provincial, sustituir importaciones, fomento de exportaciones, efecto multiplicador en la economía provincial, el respeto de normas internacionales de calidad, entre otras. Se exigió que en todos los casos el proyecto tienda "a preservar las condiciones de vida y evitar la contaminación del medio ambiente, de acuerdo a la normativa vigente". Los beneficios, a los que la agroindustria de biodiesel accedió, son: devolución de hasta un treinta por ciento $(30 \%)$ de la inversión, reintegro de hasta el 50\% o crédito fiscal a los efectos del pago de futuros impuestos por las inversiones en infraestructura (caminos, redes eléctricas, provisión de agua, desagües y otras obras), exención de tributos provinciales existentes o a crearse por un plazo de hasta diez años, entre otros.

En el momento de definir la ubicación de la fábrica, la prioridad para el grupo empresario pasaba por ubicar la molienda en el vértice sur de un cono de detección de materia prima, es decir, de la soja transgénica cultivada en el Noroeste argentino, frontera de expansión del agronegocio. Asimismo, considerando la estrategia empresarial se destacan además los siguientes factores de incidencia en decisión: a) ubicación estratégica de la ciudad, que es un punto intermedio en el tránsito de los granos desde los lugares de cultivo hacia los puertos; b) accesibilidad tanto por Ruta Nacional como por ferrocarril; c) disponibilidad de energía (redes de gas y electricidad) y d) disponibilidad de agua ya que la ciudad cuenta con un reservorio en el subsuelo (del que se abastece la empresa) y con una red de agua potable, a diferencia de la región que se caracteriza por su escasez; e) recursos humanos capacitados: la ciudad, además de ser un importante centro urbano en la zona, posee dos escuelas técnicas (TOLEDO-LÓPEZ, 2017b).

Por su parte, el proyecto empresarial fue recibido con entusiasmo por parte del gobierno local, que vio en éste el potencial para concretar objetivos tendientes a la acumulación en el plano político. La gestión tendió a viabilizar el 
emplazamiento (en articulación con la administración provincial), siendo la creación de un parque industrial funcional a los requerimientos de la empresa, el mayor indicio de esta colaboración. El proyecto de biodiesel dinamizó la concreción de la anhelada área industrial para Frías, superando rápidamente los desajustes entre la propuesta inicial del municipio y los requerimientos del grupo empresarial. El siguiente mapa muestra el lugar de emplazamiento de la empresa en el Parque Industrial. Así, las acciones de los funcionarios contribuyeron a la estrategia de reproducción del capital económico del grupo empresario, al tiempo que la propuesta de la empresa aportaba a la estrategia de los agentes gubernamentales en el campo político.

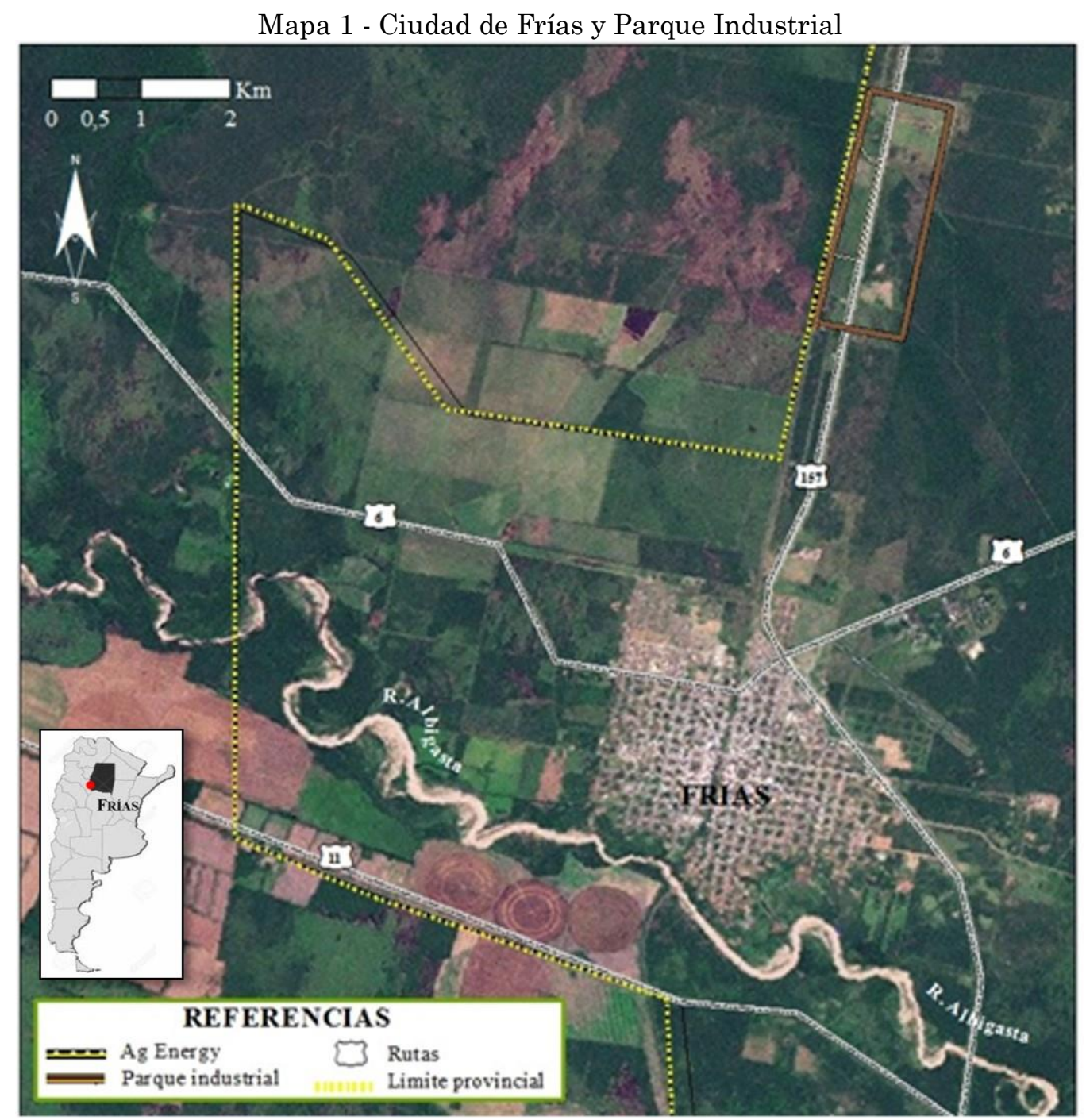

Fuente: elaboración personal.

\section{MODERNIZACIÓN ECOLÓGICA COMO MAQUILLAJE DE UN MANEJO ESTÁNDAR}

El enfoque de la "modernización ecológica" (HAJER, 1995) permeó el discurso oficial en torno del cual se articuló la narrativa del desarrollo en clave ambiental sobre la instalación de una empresa de producción de biodiesel en Santiago del Estero (TOLEDOLÓPEZ, 2016b). En principio, ello se observó en ocasión del discurso de inauguración de la fábrica a cargo de la presidenta Cristina Fernández de Kirchner, que remarcó la generación de combustibles alternativos a los fósiles como uno de los principales aportes del emprendimiento, vinculando además al evento local con las negociaciones internacionales sobre Cambio Climático y (re)instalando el eje ambiental con el que los agrocombustibles han sido promocionados a nivel nacional e internacional (PRESIDENCIA, 2009). En sus palabras: "así que, créame, no estaré en 
Copenhague, pero estoy contribuyendo con eso también a generar combustibles alternativos a los fósiles". Por otra parte, las autoridades provinciales y locales han remarcado que "es una empresa seria (...) tiene especialistas en medio ambiente, quieren hacer bien las cosas". En términos generales los relatos oficiales que dan cuenta de la responsabilidad en materia ambiental del grupo resaltan tres consideraciones:

(1) tiene un antecedente en materia de implementación de un proyecto del Mecanismo de Desarrollo Limpio (MDL) dentro del Protocolo de Kyoto porque ha sido la primer citrícola del mundo implementar el MDL con una iniciativa de recuperación del biogás a partir de los efluentes industriales (que luego es utilizado en calderas como energía térmica). De esta manera evita que se libere metano a la atmósfera y se disminuye el consumo de combustible fósil, reduciendo las emisiones de GEI. Las aguas tratadas son luego utilizadas para el riego de fincas de la misma empresa y los residuos sólidos orgánicos provenientes de plantas industriales, empaque y planta de efluentes son destinados a una playa de compostaje;

(2) las distintas certificaciones de RSE y los premios con los que cuenta el grupo: el "XIX Trofeo Internacional de Alimentos y Bebidas" recibido en 1996 en España. En 2006 La Nación y Galicia otorgaron dos premios a Vicente Lucci de "Excelencia Empresaria", premios de oro y de plata (de oro como Mejor fruticultor). En 2008 el empresario también fue seleccionado entre las 100 personalidades y organizaciones más destacadas de la década para recibir el Premio Konex "Diploma al Mérito Empresarios Rurales". Además, el grupo fue destacado por corporaciones agroalimentarias como Dow en 2007 y Coca Cola en 2008. En 2005 y nuevamente en 2010 AMCHAM (Cámara de Comercio de Estados Unidos en Argentina) otorgó al grupo (y particularmente a la Fundación) premios "Ciudadanía Empresaria" relativos al desempeño en salud y ambiente. En la temática ambiental también se destaca el premio Mercosoja 2011 por la "Metodología de cálculo para la medición de emisiones de GEI en una planta integrada de producción de biodiesel regional" y en 2012 el World Juice Awards otorgado a Citrusvil en reconocimiento a la Planta de Tratamiento de Efluentes con captación y valorización de biogás para la producción de energía térmica.

(3) La empresa ha realizado un trabajo de medición los GEI en biodiesel en coordinación con el Instituto Nacional de Tecnología Agropecuaria (INTA), considerando desde la producción primaria hasta el despacho del producto final. En el caso de la localidad de Frías los ahorros superan el $70 \%$, muy por arriba de las exigencias del 35\% del mercado europeo según la Directiva 2009/28/CE. El cálculo se basó en los datos de la empresa, incluyendo la producción en campos propios, logística, trasporte, almacenamiento, proceso industrial y flete con destino a Europa. Al respecto, se observa que el estudio fijó a inicios del 2008 el año base para la consideración sobre los cambios en el uso del suelo, quedando por fuera entonces las áreas deforestadas durante el proceso de expansión de la frontera sojera iniciada a fines de los noventa en la provincia. Asimismo, la cantidad de soja que abastece el mismo grupo que fue considerada en este informe alcanza para cubrir solo el $10 \%$ de la necesidad de soja de la planta de biodiesel (HILBERT; GALBUSERA, 2011).

Entonces, con base en estos tres elementos: participación en el MDL, certificaciones de economía verde y responsabilidad ambiental del grupo, se construyó una confianza gubernamental respecto del manejo empresarial, que interpretamos en el sentido del discurso de modernización ecológica dominante (HAJER, 1995).

Por su parte, la megaempresa se presenta con el lema "creciendo responsablemente", destacando que el "compromiso hacia la calidad y el medio ambiente se sostiene mediante nuestro sistema de gestión" (GRUPO LUCCI 2013: 19). La gestión empresarial promete tareas de control y de "monitoreo permanente" (ibid.), conforme la modernización ecológica, caracterizada por la prevalencia de un manejo preventivo y un enfoque positivo ante los problemas ambientales (HAJER, 1995). Sin embargo, la primera producción de combustible fue en junio del 2010 (destinada a abastecer el cupo nacional a través de las petroleras Refinor, Shell e YPF) sin un tratamiento ambiental de los efluentes. Las denuncias de pobladores por la muerte de animales (ver luego) plantearon dudas respecto de la inocuidad de estos líquidos.

La empresa había adquirido la tecnología Crown, de origen estadounidense, que era promocionada como de "efluentes cero", argumento que se usó para justificar el retardo en la consideración del tratamiento de los desechos líquidos del proceso industrial. Sin embargo, algunos expertos advierten que "la mayoría de las empresas que construyen plantas de fabricación de biodiesel aseguran efluente 'cero' lo cual es una utopía. Todas las Plantas de biodiesel generan efluentes líquidos, los cuales deben ser tratados convenientemente para poder 
ser volcados a los cuerpos receptores y cumplir con los parámetros de vuelco" (BELLOSO, 2011: $\mathrm{s} / \mathrm{n}$ ). Con base en la tecnología comprada por la empresa, el Estudio de Impacto Ambiental (EIA) aprobado inicialmente no consideraba a estos desechos del proceso productivo: "cuando ellos presentan la Evaluación de Impacto Ambiental se le aprueba y después cuando comienza a funcionar se ve que hay efluentes". Por su parte, el Defensor del Pueblo respondía sobre la falta de previsión respecto de los efluentes que:

dicen que es la metodología. Yo también hice la misma pregunta: por qué primero no se prevén estas cosas. Me han dicho que es imposible prever esas cosas, que en cuanto se inicia la obra monstruosa de una empresa de esta magnitud, tiene que empezar a trabajar y ver cómo va a solucionar cuando recién comiencen todas las otras tareas que se asignen en cuanto a medio ambiente. Yo también les hice la misma pregunta y me dijeron exactamente lo mismo: nosotros prevemos después de que la empresa pone en funcionamiento por primera vez. Y fue así, la verdad que fue así.

La mención a la "magnitud" de la empresa, además, reitera las consideraciones especiales que ésta ha tenido a la hora de establecerse en Frías, que justificarían, en la perspectiva de estos agentes gubernamentales que se "previera después". Ese oximorón sintetizado en la expresión "prevemos después" se distancia ampliamente de los preceptos de la modernización ecológica (HAJER, 1995). La confianza en la empresa fue fundamental en ocasión del tratamiento de problemas ambientales asociados a la producción de biodiesel, tal como se hace visible en las siguientes citas:

Es un grupo fuerte que está trabajando (...) $\mathrm{O}$ sea, le haces ver el problema y lo tratan de solucionar. En este sentido se ve la responsabilidad (...) $\mathrm{Y}$ así es que ellos en Tucumán venden bonos de carbono (...) y están certificando ISO 14000. Y aquí también la idea es llegar a certificar ISO 14000 , que son normas de calidad ambiental que son muy exigentes (funcionario, Dirección Provincial de Medio Ambiente, marzo de 2012).

No quieren tener problemas con el medio ambiente. Así que en ese aspecto estamos tranquilísimos (...) Ellos analizan permanentemente... para que ese tipo de elementos que vienen con el agua no produzcan nada con los animales ni con los humanos, porque es una empresa seria, como te dije anteriormente (entrevista al Defensor del Pueblo de la ciudad de Frías, marzo de 2012).

Así, las prácticas materiales de los actores hegemónicos estuvieron asociadas más bien al enfoque estándar de los problemas ambientales, predominando un accionar "después del hecho" (HARVEY, 1996), que se distancia de las prácticas de prevención esperadas en función del discurso de modernización ecológica dominante. En consecuencia, el grupo empresario se constituyó como el garante de hecho del manejo ambiental del proyecto que él mismo promueve a partir de las promesas de prevención y de abordaje científico-técnico de las problemáticas, siendo el enfoque de la modernización ecológica un medio para potenciar su estrategia de acumulación. Entonces, el capital económico del grupo empresario se tradujo en capital político y simbólico, relativizando el accionar empresarial al tiempo que se minimizó la advertencia de pobladores de la zona y la importancia del problema ambiental. Esto permite retomar la reflexión de Bourdieu según la cual el capital económico (definido por la riqueza detentada en relación a un sistema de relaciones económicas) constituye un "instrumento de apropiación de la dotación institucional y de los mecanismos indispensables para el funcionamiento de ese campo y, a la vez, de los beneficios que procura" (BOURDIEU, 2011: 52). En este punto es necesario también comprender el accionar empresarial en el marco de su estrategia de acumulación, de modo que la prioridad es siempre la obtención de rentabilidad alternando según le resulte conveniente entre la reducción de costos o las herramientas de la modernización ecológica vía capitalismo "verde". Por su parte, este accionar habilita la posibilidad de que los impactos y pasivos ambientales puedan pasar desapercibidos en ausencia de un conflicto social que los visibilice.

\section{CONTAMINACIÓN DE AGUAS Y DESPOJO DE ESPACIOS DE VIDA DE LA POBLACIÓN LOCAL}

Para la generación de biodiesel se mezcla alcohol y un catalizador con el aceite lográndose una reacción química conocida como transesterificación, a partir de la cual se obtiene el biodiesel y la glicerina. Los alcoholes que se 
pueden utilizar son "metanol, etanol, propanol, butanol y alcohol amílico. El metanol $(\mathrm{CH} 3 \mathrm{OH})$ y el etanol $(\mathrm{C} 2 \mathrm{H} 5 \mathrm{OH})$ son los más frecuentemente usados debido principalmente a su bajo costo y a sus adecuadas propiedades" (SORICHETTI; ROMANO, 2012: 6). Según Sorichetti y Romano (ibid.) "el metanol se elige sobre el etanol, a pesar de su alta toxicidad, debido a que el proceso de producción requiere tecnología más simple, la inversión para recuperar el exceso es de bajo costo y se logran altas velocidades de reacción" (Ibid.).

La planta de estudio utiliza metanol como alcohol y soda cáustica o hidróxido de sodio $(\mathrm{NaOH})$ como catalizador. Luego se inicia el proceso de purificación a fin de remover los contaminantes (remanentes de catalizador y metanol) de los productos de la reacción. En esta etapa se utilizan grandes cantidades de agua, por lo que es primordial un uso eficiente y la implementación de técnicas para su recuperación. El desperdicio de agua para las plantas de gran tamaño ha sido estimado en un $35 \%$, en relación al volumen de biocombustible a purificar (SORICHETTI; ROMANO, 2012: 3).

En el caso de estudio según datos provistos en el sitio web de la empresa (recuperado en julio de 2017) "las aguas residuales con alta carga orgánica" se proyectan en $600 \mathrm{~m} 3 /$ día. Tal como afirma una investigación reciente en la materia "en esta etapa compuesta por sucesivos lavados con agua, se generan grandes volúmenes de aguas residuales, que supondrían graves daños medioambientales si fueran devueltas al medio sin recibir tratamiento previo" (ROJO-CHOYA, 2015: 7). Un informe publicado en por la Comisión Económica para América Latina y el Caribe (CEPAL) señala que "los principales problemas ambientales asociados a la producción de aceites vegetales son la generación de residuos sólidos con una alta Demanda Bioquímica de Oxigeno (DBO) y la presencia de malos olores" (GÓMEZ-GARCÍA et. al., 2008: 20). Según los autores, "pueden producirse emisiones marginales de metanol por venteo y de óxido de azufre". En el caso estudiado también se denunció el mal olor.

Respecto a vertidos en cuerpos de agua, el de la producción de bio o agrodiesel es un proceso que genera aguas aceitosas y jabonosas, dependiendo de la eficiencia del separador de aceites (MARTICORENA et al., 2010). Cuando los residuos líquidos contienen mucha materia orgánica los niveles de oxígeno disuelto disminuyen y esto afecta negativamente a peces y otros organismos acuáticos. También el $\mathrm{pH}$ y el contenido de residuos de metanol de estos efluentes deben ser controlados dados los riesgos ambientales de contaminación de aguas.

Tal como muestra el trabajo de Rojo-Choya (2015) los efluentes de la producción de biodiesel arrastran las impurezas contenidas en la mezcla de ésteres metílicos (principalmente trazas de grasas, aceites, catalizador básico, sales, jabones, trazas de glicerol y metanol), con una elevada carga contaminante. También Sorichetti $\mathrm{y}$ Romano (2012) afirman que los efluentes de la producción de biodiesel entrañan riesgo ambiental de contaminación en tanto el agua de lavado contiene cantidades variables de alcohol y catalizador. Así, en palabras de los especialistas "involucra serios riesgos ambientales debido a la contaminación de las napas de agua subterránea, cursos de agua y sistemas de desagüe (en áreas urbanizadas)" (SORICHETTI; ROMANO, 2012: 8). En consecuencia, un tratamiento deficiente de los efluentes del biodiesel implica serios problemas ambientales, que pueden derivar en disputas por el acceso y uso de los bienes comunes naturales acrecentando la conflictividad territorial.

En el caso de estudio al iniciar la producción de combustible se dio curso a los efluentes a través de canales preexistentes que servían de contención ante las crecidas de un río cercano:

un canal permanente que está hecho porque hay un viejo río que viene de una zona de Catamarca que está encausado (...) porque tuvimos una enorme inundación en la ciudad, entonces ese canal es un canal que en ese caso, si vuelve a haber una inundación, deriva el agua que sale al costado de la ciudad y evita que entre a la ciudad. [Con la cuestión del efluente] entonces se abrió, se limpió el canal (entrevista al Director de Medio Ambiente Provincial, marzo de 2012).

El mapa 2 permite observar el Parque Industrial de Frías y la disposición de los canales a cielo abierto. Se señalan además las zonas donde fueron realizados registros fotográficos que documentaron la disputa ambiental referida a continuación. 
Mapa 2 - Parque Industrial de Frías y zona de conflicto

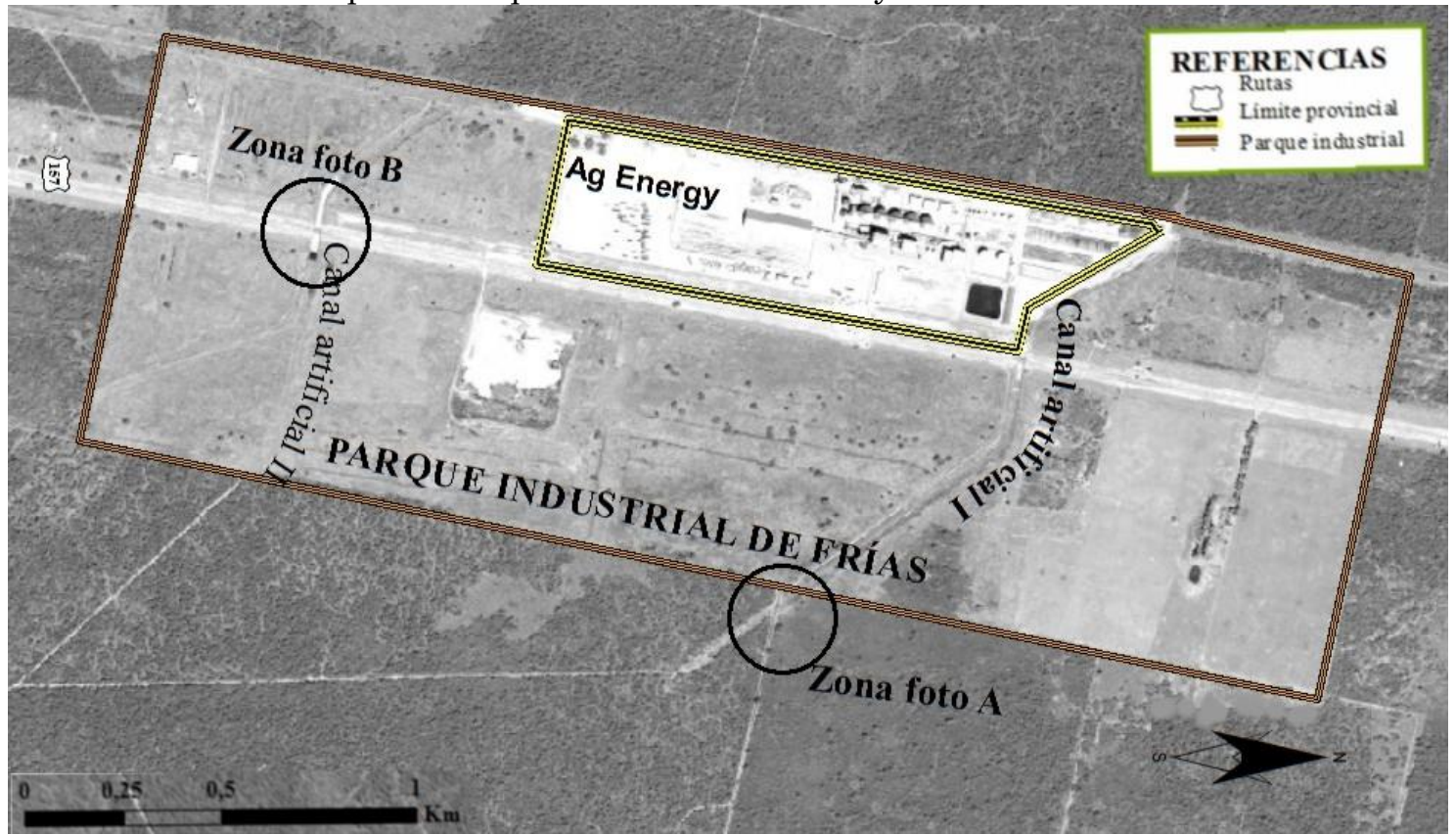

Fuente: elaboración propia en base a imagen satelital de Google Earth año 2010.

En relación al contenido de estos efluentes, otra vez se observa "confianza" de parte de las autoridades estatales con el manejo empresarial. Desde el municipio se decía:

La planta empezó a procesar y arroja al canal agua de caldera, 18 mil litros de agua de caldera, que se arrojan con productos del lavado del proceso. $\mathrm{O}$ sea, ¿qué es el producto del lavado del proceso? Aceite, algo de aceite de soja, que es biodegradable, y trazas de harina, o sea que la empresa si tira mucho de eso está perdiendo dinero. Como eso es un producto orgánico y esa proteína tiene el mismo olor que la carne en descomposición, porque cuando se moja la proteína, se descompone y tiene ese olor (entrevista al Secretario de Producción del Municipio de Frías, marzo de 2012).

El razonamiento del Secretario de Producción se basa en la asociación "lógica" según la cual, al ser el combustible de origen orgánico, el desecho "debiera ser" inocuo, desconociendo el proceso industrial a través del cual se obtiene el biodiesel y las evidencias respecto de la contaminación del mismo, así como también las denuncias de los pobladores en relación a los animales muertos por beber agua del canal a cielo abierto.

Los primeros reclamos ambientales formales se realizan a principios del 2011 y la vía elegida para encauzarlos fue la denuncia ante el Defensor del Pueblo de la ciudad de Frías para que interceda ante la empresa. Es preciso destacar que al indagar sobre el proceso desencadenado el Defensor inició su relato advirtiendo que

una empresa que tiene 100 millones de dólares de inversión es indudable que algún problemita va a tener. Pero no obstante eso nosotros estamos contentos por lo que está dándole a la ciudad de Frías, como una empresa más grande (...) es un orgullo para Santiago del Estero y es un orgullo para la ciudad de Frías (entrevista al Defensor del Pueblo de la ciudad de Frías, marzo de 2012).

Tras reafirmar entonces su percepción sobre la relevancia de la empresa y justificar una moderación en los problemas acontecidos en función de características del emprendimiento (tamaño de la planta y monto de la inversión), describe que las denuncias provinieron de un grupo de pobladores "de alrededor de la ciudad de Frías" y que versaron

más que nada sobre los efluentes que la empresa envía a través de (...) un canal. Esos efluentes empezaron a hacer unos recorridos, más o menos como de $10 \mathrm{~km}$. y comenzó a haber un olor félido, moscas, un color viscoso amarillo (entrevista al Defensor del Pueblo de la ciudad de Frías, marzo de 2012).

A partir de la denuncia, la Defensoría acudió 
al lugar para observar e iniciar las estipuladas por el protocolo. Según el representante gubernamental el accionar llevado a cabo consistió en cumplir con los procedimientos estipulados "para que la Defensoría se cubra en cuanto a su trabajo". Así, las tareas llevadas a cabo fueron:

llegamos a la zona, sacamos fotografías y enviamos y comenzamos a hacer las denuncias correspondientes. Una vez que realizamos nuestra tarea (...) hemos empezado a dialogar con la empresa para que este tipo de situaciones en el futuro no sigan. Tal es así que a través de notas que mandamos nosotros, y la visita de ellos, nos hicieron un informe correspondiente [sobre] qué es lo que ellos están haciendo a futuro. Entonces, están trabajando en la planta en las piletas para que esos efluentes no salgan tal y cual como están hoy día (entrevista al Defensor del Pueblo de la ciudad de Frías, marzo de 2012).

Una vez desplegado el conflicto en el ámbito público y habiendo sido encauzado el reclamo por las vías formales, la empresa firmó un "Acuerdo de Producción Limpia" con las autoridades de la Provincia de Santiago del Estero cuyo objetivo es "la adecuación progresiva y constante a la normativa ambiental vigente y la promoción e implementación de Buenas Prácticas de Gestión Ambiental". Dentro del Acuerdo, la empresa se comprometió a construir una Planta de Tratamiento de efluentes en tres etapas. Con este fin el 14 de junio de 2011, la Corporación Interamericana de Inversiones (CII) aprobó un préstamo de hasta 40 millones de dólares, en dos partes, a Viluco S.A. Como contrapartida la empresa se comprometió a elaborar un Plan de Gestión Ambiental (PGA) para asegurar el cumplimiento de la normativa nacional y las pautas de la CII con respecto a los aspectos medioambientales y de seguridad e higiene laboral.

En el momento del trabajo de campo (año 2012) la etapa 1 ya se encontraba en funcionamiento (realizando una separación primaria), y las fases 2 y 3 estaban en construcción. Los funcionarios públicos remarcaban en sus relatos el cumplimiento por parte del grupo empresario con la planificación acordada, como forma de resguardar el prestigio y la imagen que se transmiten de la empresa, y defendiendo así las expectativas (de desarrollo) en relación al proyecto:

están trabajando en la planta en las piletas para que esos efluentes no salgan tal y cual como están hoy día. Que simplemente sea agua limpia. Ese sería el final de nuestra lucha para que no haya contaminación en la ciudad y mucho menos en la zona. Ellos entregaron un informe técnico (de) cuáles son los trabajos que se van a hacer en el primer año, cual es el trabajo que se va a hacer en el segundo año y cuales en el tercer año (...).

Permanentemente ellos me están informando qué es lo que están haciendo. Me invitaron yo fui dos veces a la planta, vi las obras que están haciendo y están super adelantados. Y es una empresa seria, hasta el momento me han demostrado honestidad en sus actos y que en el futuro va a terminar con este tipo de contaminación ambiental" (entrevista al Defensor del Pueblo de la ciudad de Frías, marzo de 2012).

Sin embargo, pese a que el Defensor del Pueblo marca el cumplimiento del acuerdo como punto final de la disputa ambiental existente en relación a los efluentes y los olores, esa resolución no satisfizo la voluntad de algunos vecinos que demandaban dejar de ver afectada su territorialidad. Para los afectados el problema persistía porque aun cuando se estaban ejecutando las obras de construcción de las piletas de tratamiento, la empresa continuaba volcando efluentes a cielo abierto y sin tratamiento. Ello derivó en un incremento de la conflictividad en algunos casos, tal como ilustra la fotografía, correspondiente a la "zona foto A" indicada en el Mapa 2. En ese caso, ante la falta de respuesta de las autoridades y el hecho de haber "dejado de ser amigos" con la empresa tras sus reiteradas quejas, el vecino decidió realizar una presa al borde del alambrado de su propiedad impidiendo de esta manera el ingreso de los efluentes de la planta de biodiesel. 


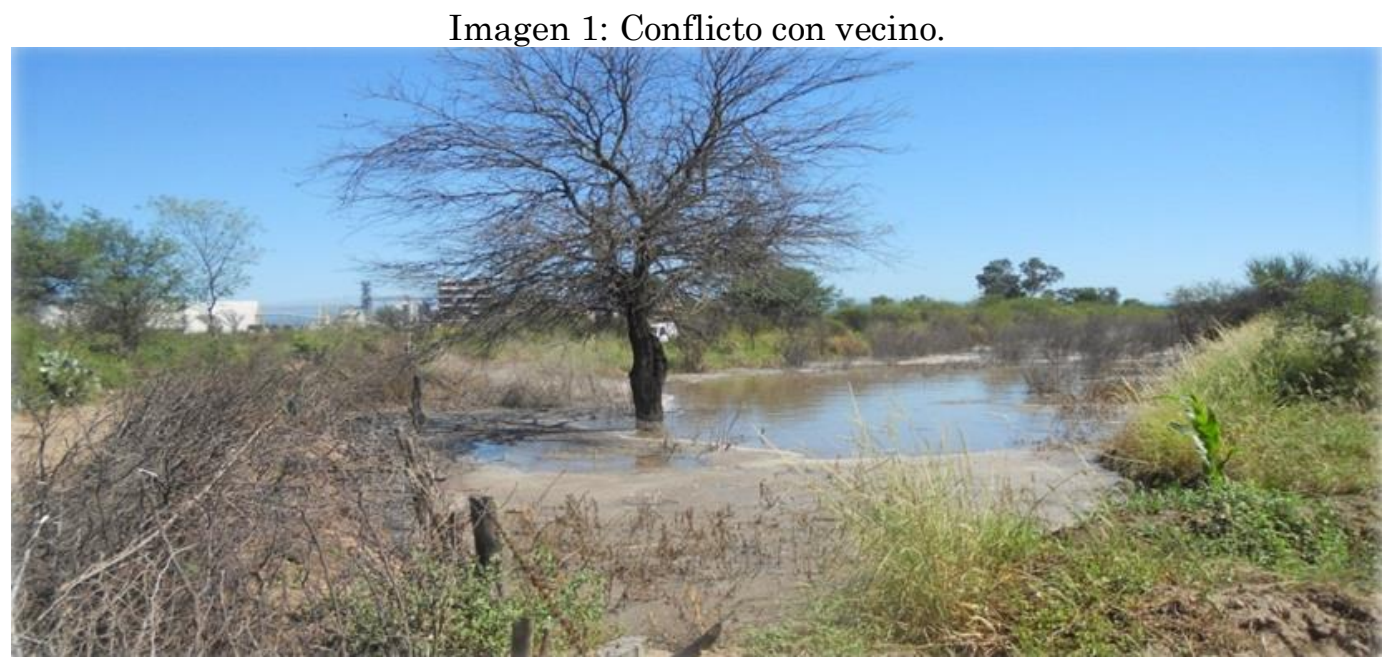

Fuente: archivo personal, marzo de 2012. La imagen refleja la medida adoptada por un vecino de la planta de biodiesel, al que el canal a cielo abierto le atravesaba su campo.

La imagen 1 muestra el efluente estancado producto del cerco generado por el vecino al lado del alambrado, y al fondo a la izquierda puede observarse la silueta de la planta de biodiesel en el horizonte. Frente a este proceder, las autoridades tomaron medidas para revertir esa situación, en detrimento del interés del afectado:

A veces tenemos problemas y se taponean los canales. Yo tuve que hacer una presentación en la justicia, logré de nuevo que se abran los canales para que corra el agua, porque nosotros tomamos el canal como un canal público que necesita de alguna manera que (...) para que corra cualquier tipo de agua, ya sea de creciente de lluvia o en este caso del efluente. Y en el futuro se está hablando de que esto se va a canaletear ... y ahí terminamos, pero tiene su tiempo, nosotros somos comprensivos, la empresa está trabajando en eso, está muy bien (entrevista al Defensor del Pueblo de la ciudad de Frías, marzo de 2012).

Ante estos acontecimientos los funcionarios públicos remarcan, una vez más, el tipo de inversión, la generación de trabajo y el capital social objetivado del grupo empresario:

Este tipo de empresas indudablemente que han generado un poquito más de movimiento (...) Es un monto muy grande que van a dedicar, millones de dólares, en cuanto al tratamiento de este tipo de agua (...) eso es lo que a mí me llena de orgullo para la ciudad de Frías. No es una empresa fantasma que vino a hacer plata. No, es una empresa que vino a plantar, a trabajar, a dar posibilidades a la gente y que ellos ganan dinero (...). Pero es una empresa seria (...) Esta es una empresa líder" (entrevista al Defensor del Pueblo de la ciudad de Frías, marzo de 2012)

Así, se exige tolerancia a los pobladores que ven afectado su tradicional uso del espacio, por un objetivo superior (el "desarrollo") que propicia la "empresa líder". No obstante, se reconoce la importancia de que exista esa "vigilancia" social al tiempo que se relativiza la opinión pública, planteando que es necesario "explicarles" el porqué del daño generado, tomando sus críticas para resolver en función de "lo posible":

Muchas de las denuncias son infundadas, pero hay que verlas. Y hay que ir a la gente también. (...) Si vos le explicas cómo es, qué es lo que está pasando... lo aceptan, o no, pero oralmente lo aceptan si vos le explicas bien, lo aceptan, o sea, tienes que demostrarles. Y, vuelvo a decirte, mucha de las cosas la gente no denuncia porque no conoce, y cuando comienzan a conocer hacen la denuncia y se soluciona en partes, lo posible (entrevista al Director General de Medio Ambiente, Secretaría del Agua del Gobierno de la Provincia de Santiago del Estero, marzo de 2012).

Esta misma idea de qué es lo posible y lo deseable, y de que es preciso "sacrificar" algunos objetivos de cuidado ambiental en pos del "desarrollo" es un razonamiento que se reitera en las narrativas de los funcionarios entrevistados. De esta manera, no se buscan maneras de revertir los daños observados sino más bien asumiendo el statu quo, arribar al "mal menor". En palabras del Director General de Medio de la 


\section{Provincia:}

Uno no puede decir no a los combustibles fósiles. Tiene que seguir, pero siempre buscando (...) De todas maneras la producción de diesel sirve para minimizar, o para disminuir los combustibles fósiles, el consumo de combustibles fósiles.

En ese razonamiento, los contaminantes asociados a la producción de biodiesel son un "mal menor" en relación tanto al uso de combustibles fósiles como a las ventajas derivadas de la industrialización. Se asume entonces que la contaminación es el costo que hay que sacrificar en pos del trabajo y el desarrollo. Así, el responsable de Medio Ambiente afirma:

en la parte personal te digo que ... preferiría que a la soja la hagan comida no diesel pero de todas maneras, eso es personal no? Y bueno, para mí todo lo que haga a la transformación de materia prima es lo mejor. O sea, lo peor que podemos hacer es vender la materia prima sin ningún tipo de transformación (...) El único problema es, siempre, bueno vas a tener la parte de contaminación, eso que hay que tratar de minimizar. Pero siempre es mejor vender productos y no materia prima" (Ibíd.)

El testimonio del funcionario expresa una visión en la que la cuestión ambiental se plantea en oposición a la industrialización y el empleo. Este relato asume el camino hacia el desarrollo linealmente, planteando que sólo es posible elegir entre un número limitado de opciones, y tomando en este marco "soluciones de compromiso" que expresan el "mal menor", aunque se perciba que serían "preferibles" otras/nuevas experiencias. Por otra parte, también da cuenta que pese a los (nuevos) estándares que regulan la actividad económica, la relación naturaleza-sociedad continúa planteándose para algunos agentes gubernamentales en términos productivistas y bajo un paradigma en el que las metas de la economía y de la ecología se oponen.

Por su parte, frente a los reclamos la empresa desplegó distintas estrategias haciendo uso del respaldo de los organismos del Estado (mecanismos de dominación indirectos) y utilizando también mecanismos de dominación directos allí donde los indirectos no alcanzaron. Estos últimos consisten, según Bourdieu (2011: 43), en "relaciones de dependencia personal", "fundada en la dependencia material". Ilustrativo de estas prácticas resulta el testimonio de una vecina obtenido durante el trabajo de campo. Ella señaló que desde que estaba en funcionamiento la planta algunos de sus animales se habían muerto por beber agua del canal, además de sentir molestias por el olor nauseabundo que emanaba la empresa. Sin embargo, había decidido no formalizar una denuncia porque "se apareció una de las dueñas de la fábrica" para decirle que ella estaba "permisada" a visitar la planta cuando quisiera, y a "solicitar alimento para sus animales", mientras le dejaba de regalo descartes de la soja. Así, la vecina agradecida por este gesto de la empresaria decidió no estropear ese vínculo, asumiendo al mismo tiempo la distancia y las jerarquías sociales. En este punto es preciso no perder de vista la advertencia sobre la lógica de las prácticas que hiciera Bourdieu (2011: 69), según la cual en algunos casos "el modo de dar vale más que aquello que se da", reafirmando mediante una "conducta generosa" las relaciones de fuerza.

\section{CONSIDERACIONES FINALES}

La producción de biodiesel en Santiago del Estero se comprende como parte del proceso de expansión del agronegocio en la región, siendo esta agroindustria un modo de reafirmación mediante el "agregado de valor" de la soja transgénica generada en el NOA. El caso muestra el despliegue de una territorialidad que implica un uso funcional del espacio por parte de la empresa, sustentada en una posición de jerarquía derivada de su capital económico, traducido en capital social, simbólico y político. Este proceso entraña una conflictividad cuyos elementos estructurantes se vinculan con la intromisión de un agente cuyo uso del espacio entra en conflicto con los usos (y significados) que la población tenía del mismo. Resulta así un ejemplo de las contradicciones existentes entre el espacio económico del agronegocio y el espacio de vida de la población local, cuyo desarrollo da cuenta del desafiante predominio de la lógica del primero. Así, el caso ejemplifica cómo las disputas por el uso del ambiente ponen en evidencia las relaciones de poder que estipulan la distribución de los recursos en juego, lo que implica para unos la capacidad de decisión sobre su utilización y para otros la exclusión de su disponibilidad y disfrute.

Por su parte, el conflicto da cuenta de 
dimensiones desatendidas durante el proceso de implantación, pese al discurso de responsabilidad social y ambiental empresarial predominante. El estudio permitió observar que tanto el accionar de la empresa como de los agentes gubernamentales se distancia de las prácticas de prevención que se hubiesen esperado de acuerdo con el uso del discurso de modernización ecológica. En particular, en la disputa en relación a los efluentes, los contaminantes asociados a la producción de biodiesel se concebían como un "mal menor" y se asumía la contaminación como el costo a sacrificar en pos del trabajo y el desarrollo.

La observación del caso de Frías muestra que el accionar empresarial prioriza la obtención de rentabilidad sobre cualquier otro objetivo de índole social, ambiental, cultural, etc., ocupándose de éstos toda vez que sea requisito para reproducir la acumulación (incorporando herramientas de la modernización ecológica vía capitalismo verde o bien reorganizando los costos, el empleo y las inversiones). Por su parte, el capital social objetivado y el capital simbólico del grupo empresario le permitieron "maquillar" su accionar, relativizando las demandas ambientales y la importancia de los problemas acaecidos. Por otro lado, se destaca el rol facilitador que desplegaron los funcionarios públicos sosteniendo una actitud "comprensiva" para con los tiempos y a la voluntad de la empresa, mientras que frente a las protestas sociales se intenta su disuasión (especialmente frente a las que poseen el potencial de perturbar la narrativa construida en torno a la empresa). Así, pese al discurso dominante de la modernización ecológica, las prácticas materiales de los actores hegemónicos continúan asociadas a un enfoque no preventivo y estándar de la gestión ambiental, siendo el cuidado de los bienes comunes naturales subsidiario de la acumulación de capital.

Finalmente, el caso de estudio da cuenta de la productividad del planteo de la disputa en el ámbito público, en tanto exige medidas tendientes a mejorar la gestión ambiental, observando los procesos productivos a fin de disminuir los impactos en el medio. La disputa ambiental desafió el statu-quo, demandando nuevos posibles, mostrando la necesidad de revisar las prácticas existentes en aras del bienestar socioambiental. Por lo demás, también el conflicto habilita un ejercicio de reflexión social que se mantiene abierto, en torno a discursos que tienden a presentarse como cerrados y lineales.

\section{AGRADECIMENTOS}

La presente investigación fue posible gracias a la beca doctoral otorgada por el CONICET y a la colaboración de los y las habitantes de la ciudad de Frías.

\section{REFERENCIAS}

ARGENTINA, Ley 26093, disponible en <http://www.infoleg.gov.ar/infolegInternet/> Acceso 20 de enero de 2020.

AZUELA, A.; MUSSETTA, P. Algo más que el ambiente. Conflictos sociales en tres áreas naturales protegidas de México, Revista de Ciencias Sociales. Segunda Época 1, v. 16, 2005, p. 191-206.

BELLOSO, C. Tratamiento de efluentes líquidos en la industria del Biodiesel, Ponencia presentada en el Encuentro para la Industria del Biodiesel, Centro de Ingenieros de Rosario y la Asociación de Ingenieros Químicos de Rosario, 2 y 3 noviembre de 2011, Rosario.

BORRAS J.R., SATURNINO M.; MCMICHAEL, P; SCOONES, I. The politics of biofuels, land and agrarian change. The Journal of peasant studies, 2010 , v.. 37, n 4, p. 575-592.

BOURDIEU, P. Las estrategias de la reproducción social, Siglo XXI, Bs As., 2011. CARRIZO, S. C., GUIBERT, M.; BERDOLINI, J. Actores y mercados de los
biocombustibles incertidumbre y diversificación. Ponencia presentada en el 12do Encuentro de Geógrafos de América Latina - EGAL, 03 al 07 de abril de 2009, Montevideo, Uruguay.

CII - Corporación Interamericana de Inversiones, Documento del Directorio Ejecutivo. Acta de la reunión del 14 de junio de 2011 aprobada el 3 de agosto de 2011. Disponible en <http://idbdocs.iadb.org/wsdocs/getdocument.as px?docnum $=36326603>$, Acceso el 1 de abril de 2015.

GIARRACCA N., TEUBAL, M. (Comp.) Actividades extractivas en expansión ¿Reprimarización de la economía argentina?, Antropofagia, Bs. As.: 2013.

GÓMEZ GARCÍA, J.; SAMANIEGO, J. L.; ANTONISSEN, M. Consideraciones ambientales en torno a los biocombustibles líquidos, CEPAL (Serie Medio Ambiente y Desarrollo) 137, 2008.

GOODMAN, L. A. Snowball sampling. The 
annals of mathematical statistics, 1961, p. 148 170.

GUTIÉRREZ, A. Las prácticas sociales: una introducción a Pierre Bourdieu, Ferreyra Editor, Córdoba, 2005.

GRUPO LUCCI, Memoria de Sustentabilidad, 2013, Disponible en: <https://docplayer.es/15245118-Grupo-luccimemoria-de-sustentabilidad-2013-seccioninicial.html> Acceso el 20 de énero de 2020.

HAJER, M. The politics of environmental discourse, Clarendon Press, Oxford, 1995.

HARVEY, D. Justice, nature, and the geography of difference, Blackwell, Oxford. 1996

HILBERT, J.; SBARRA R.; LÓPEZ-AMORÓS, M. Producción de biodiesel a partir de aceite de soja. Contexto y Evolución Reciente, Ediciones INTA, 2012.

;GALBUSERA, S. Análisis de emisiones. Producción de biodiesel - Ag Energy, Documento INTA, 2011, Disponible en: $<$ http://inta.gob.ar/documentos/analisis-deemisiones-produccion-de-biodiesel-2013-agenergy/> Acceso el 1 de marzo de 2014.

ÍNIGUEZ-RUEDA, L. Análisis del discurso: manual para las ciencias sociales. Universitat Oberta de Catalunya, Barcelona, 2006.

INDEC - Instituto Nacional de Estadísticas y Censos, Arg., Biocombustibles. Primer trimestre de 2016, 2016; Disponible en: http://www.indec.gov.ar/uploads/informesdepre nsa/biocombustibles_05_16.pdf, Acceso el 1 de mayo de 2017.

MARTICORENA, A. A., MANDAGARÁN, B. A.; CAMPANELLA; A. Análisis del Impacto Ambiental de la Recuperación de Metanol en la Producción de Biodiesel usando el Algoritmo de Reducción de Desechos WAR, Información Tecnológica 1, v. 21, 2010.

MERLINSKY, M. G. (Comp.) Cartografias del conflicto ambiental en Argentina (I), CICCUS-CLACSO, CABA, 2013.

MINISTERIO DE HACIENDA, Informes cadenas de valor, 2017. Disponibles en <https://www.economia.gob.ar/peconomica/docs/ 2017/SSPMicro_Cadena de Valor Oleaginosa. pdf>, Acceso 20 de enero de 2020.

OCDE/FAO - Perspectivas Agrícolas 20172026, OECD Publishing, París; 2017. DOI: <http://dx.doi.org/10.1787/agr_outlook-2017-13es> Acceso 20 de enero de 2020.

PANICHELLI, L. Análisis de Ciclo de Vida (ACV) de la producción de biodiesel (B100) en Argentina. Tesis (Lic. Agronomía de la UBA), Buenos Aires, 2006.

PIMENTEL, D.; PATZEK, T. Ethanol production using corn, switchgrass, and wood; biodiesel production using soybean and sunflower, Natural Resources Research 1 v. 14, 2005, p. 65-76.

PRESIDENCIA, Discurso presidencial, Parque industrial de Frías, Santiago del Estero, 16 de diciembre de 2009, disponible en <http://www.presidencia.gob.ar/discursos/3683 $>$, Acceso 10 de diciembre de 2012.

REGUILLO, R. De la pasión metodológica o de la (paradójica) posibilidad de la investigación, En MEJIA-ARAUZ, R.; SANDOVAL, A. (coord.): Tras las vetas de la investigación cualitativa. Perspectivas y acercamientos desde la práctica, ITESO, México, 1998.

REYES O. (ed.) Preparando el terreno para los agrocombustibles: políticas europeas, criterios de sostenibilidad y cálculos climáticos, 2007, Disponible en: <http://www.biodiversidadla.org/> Acceso $28 \mathrm{de}$ abril de 2008.

ROJO-CHOYA, M. Planta de tratamiento de aguas residuales procedentes de la producción de biodiesel. Tesis (Lic. Ingeniería Química, Universidad de Valladolid, España), 2015.

ROZEMBERG, R.; SASLAVSKY, D.; SVARZMAN, G. La Industria de Biocombustibles en Argentina, en LÓPEZ A. (coord.): La Industria de Biocombustibles en el Mercosur (Serie Red Mercosur) v. 15, 2009.

RUNGE, C. F.; SENAUER, B. How biofuels could starve the poor. Foreign Aff., v. 86, 2007, p. 41.

SANTIAGO DEL ESTERO, Ley Provincial de Promoción Industrial, 6.750, disponible en $<$ http://www.dgrsantiago.gov.ar/wpcontent/uploads/2017/11/LEY-6750.pdf> acceso 01 de enero de 2020.

SAUTU, R., BONIOLO, P., DALLE, P.; ELBERT, R. Manual de metodología: construcción del marco teórico, formulación de los objetivos y elección de la metodología. CLACSO, Bs. As., 2005.

SCHEINKERMAN DE OBSCHATKO E.; BEGENISIC, F. Coords. Perspectivas de los biocombustibles en la Argentina y Brasil, SAGPyA/IICA, Buenos Aires, 2006.

SMOLKER, R.; TOKAR, B., PETERMANN, A.; HERNÁNDEZ. E. El verdadero costo de los agrocombustibles. Alimentación, bosques y clima, 2007, Disponible en: <http://www.globalforestcoalition.org/newsandp ublications/publications/> Acceso 9 de mayo de 2008.

SORICHETTI, P. A.; ROMANO, S. Uso de agua 
en la purificación de biodiesel: optimización mediante el control de propiedades eléctricas de efluentes, Ponencia presentada en VII Congreso de Medio Ambiente-AUGM, 22 al 24 de mayo 2012, UNLP, La Plata.

TOLEDO-LOPEZ, V. Agroenergía en Argentina: una discusión sobre la renovabilidad y el despojo, GUTIERREZ, F. (ed.): Soberanía energética, propuestas y debates desde el campo popular, Ediciones del Jinete Insomne, CABA, 2018, p. 117-147. Disponible en: < < <tp://www.opsur.org.ar/blog/wpcontent/uploads/2018/12/Libro-Soberaniaenergetica-WEB.pdf $>$, Acceso 20 de enero de 2020.

TOLEDO-LOPEZ, V. La política agraria del kirchnerismo. Entre el espejismo de la coexistencia y el predominio del agronegocio. Mundo Agrario 37, v. 18, 2017a, DOI: https://doi.org/10.24215/15155994e045.

TOLEDO-LOPEZ, V. Territorio del agronegocio. Estrategias de acumulación y lógicas de territorialización de las megaempresas. Reflexiones a partir de un estudio de caso en el NOA argentino. Trabajo y Sociedad 29, 2017b, p. 177-19, Disponible en <http://www.redalyc.org/articulo.oa?id=387352 $369010>$

TOLEDO-LOPEZ, V. Desarrollo y agroenergía. Un análisis de narrativas regionales y locales a propósito de la producción de biodiesel en Santiago del Estero, MERLINSKY, G. (Comp.) Cartografías del conflicto ambiental en Argentina (II), CICCUS-CLACSO, CABA, 2016a.

TOLEDO-LOPEZ, V. Naturaleza del agro y sentidos sobre el desarrollo. Un estudio sobre los procesos de legitimación de la agroenergía a partir del caso de la planta de biodiesel de Santiago del Estero. Tesis (Doctorado en ciencias sociales, FCS-UBA), $2016 b$.

TOLEDO-LOPEZ, V. Los agrocombustibles como eje del extractivismo en la Argentina, GIARRACCA N.; TEUBAL, M. (Comp.) Actividades extractivas en expansión ¿Reprimarización de la economía argentina?, Antropofagia, Bs. As.: 2013. 